\title{
Charity focus
}

\section{Oral health for the homeless}

Henry Schein Dental UK has partnered with the British Dental Industry Association (BDIA), and Crisis, the national charity for homeless people, for the ninth consecutive year to provide emergency oral health treatment to more than 730 homeless people in London (one is pictured). Crisis for Christmas served 38,000 meals in London throughout the holiday week and more than 5,600 meals across centres on Christmas day. More than 11,000 volunteers contributed approximately 250,000 volunteering hours.

\section{Fundraising in Mumbai}

The managing director of dental IT solutions provider Microminder has run the Mumbai half marathon to raise funds for international dental charity Dentaid.

Bharat Sheth joined 43,600 runners in the event on 22 January and raised $£ 1,800$ along the way. After a 5.40 am start to beat the intense heat, Bharat, 57 , completed the race in just over three hours.

Bharat was fundraising for Dentaid's mobile dental unit which will visit homeless shelters, day centres and schools to provide dental treatment for vulnerable people who find it difficult to access dental care and oral health education for children.

\section{Local training in Africa}

Volunteers from the UK spent two weeks in remote parts of Tanzania, training rural clinical officers to safely extract teeth and treating hundreds of patients. The programme was organised by Bridge2Aid and supported by mydentist.

\section{Brushing in Leeds}

Children in Leeds have been learning how to look after their teeth thanks to the Bright Bites project run by Dentaid. Volunteer dentist Nick O'Donovan is visiting schools in the city to teach the children about good tooth brushing, the amount of sugar in popular food and drinks and the importance of regularly visiting the dentist. The project is being funded by the People's Postcode Lottery which gave Dentaid a $£ 9,100$ grant to fund its oral health education work in Leeds. In addition to the presentation all the children receive a tooth brushing chart, recipes, low sugar snack suggestions, a tube of toothpaste and a toothbrush. Bright Bites also includes classroom activities

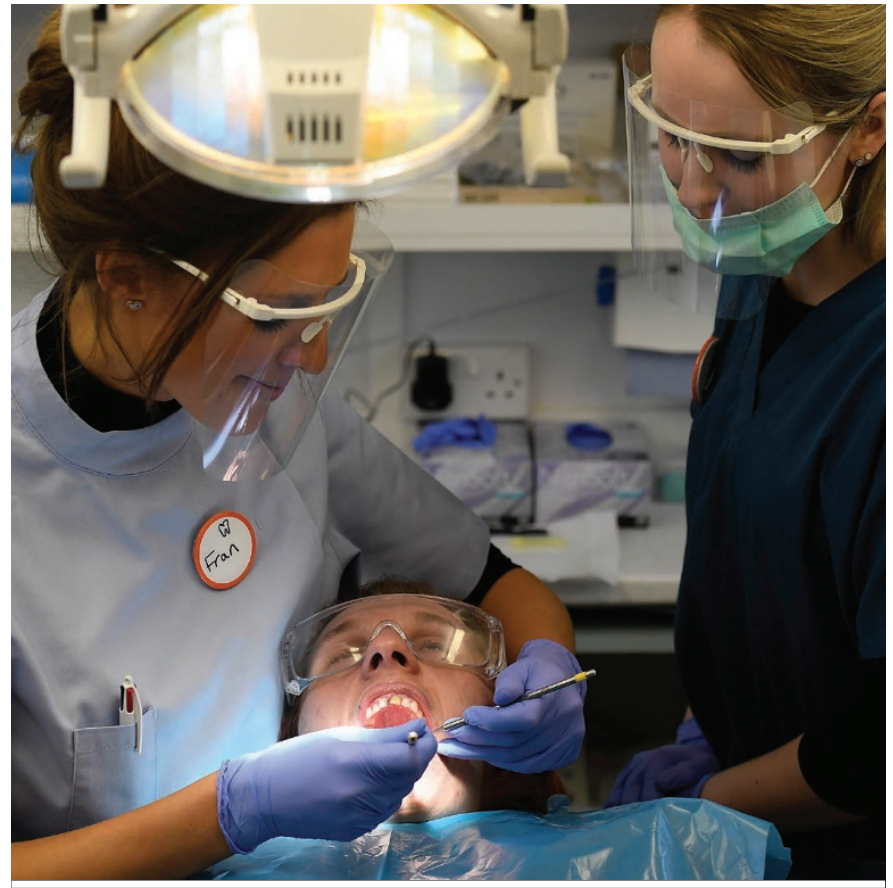

Henry Schein Dental UK and the BDIA joined with Crisis in 2016 to provide emergency oral health treatment to more than 730 homeless people in London

with experiments showing the damage that sugar can do along with resources and lesson plans for teachers; parents are also invited to Dr O’Donovan's presentation.

\section{Volunteers needed for Zimbabwe}

Dentaid is offering two dental professionals the chance to join its volunteering team in the beautiful country of Zimbabwe from 15 to 29 October 2017.

Working alongside local dental professionals, the team will visit schools, an orphanage, a prison, an HIV clinic and community centres in the region of Chinhoyi. The volunteering trip will end with two days at the Victoria Falls.

Dentists, dental nurses, hygienists and therapists are all invited to apply for the two week volunteering trip. Volunteers are asked to raise $£ 2,250$ to cover and fundraising support is available. Visit https://dentaid.org/missions/volunteer-zimbabwe/.

\section{Calling all Manchester alumni}

The SOciety of Manchester Dental Alumni (SOMANDA) are holding their 30th Anniversary Biennial Reunion on Friday 31 March 2017.

The formal meeting and lectures will take place in the MANDEC postgraduate suite at the Manchester Dental School. Manchester staff alumnus Professor Trevor Burke, currently Professor of Primary Care, Birmingham, will provide the FC Wilkinson Commemorative lectures. Two hours of verifiable CPD are available. The Dean of School Professor Paul Coulthard will give an update of the School and there will be an opportunity to tour the clinics. Following on from the scientific meeting the evening will be rounded off at Christie's Bistro in the Whitworth Building with a dinner and plenty of opportunity to catch up with fellow alumni.
SOMANDA supports the school with student prizes, funds to assist with student hardship and overseas study costs. They also offer a CPD bursary allocated by a prize draw among members for use in support of verifiable CPD.

All alumni are invited and SOMANDA hope many will consider attending this year.

Please contact Julie Wright on thisisjmw@gmail.com. 